\title{
Effectiveness and safety of intracoronary papaverine, alprostadil, and high dosages of nicorandil and adenosine triphosphate for measurement of the index of coronary microcirculatory resistance in a pig model
}

\author{
Tianbing Duann ${ }^{1,2, A-F}$, Jinxia Zhang ${ }^{2, B}$, Dingcheng Xiang ${ }^{2, A, E}$, Rui Song ${ }^{2, C}$, Ranran Kong ${ }^{2, C}$, Dingli Xü ${ }^{1, A, F}$ \\ 1 Department of Cardiology, Nanfang Hospital, Southern Medical University, Guangzhou, China \\ 2 Department of Cardiology, Guangzhou General Hospital of Guangzhou Military Command, China \\ A - research concept and design; $\mathrm{B}$ - collection and/or assembly of data; $\mathrm{C}$ - data analysis and interpretation; \\ $\mathrm{D}$ - writing the article; $\mathrm{E}$ - critical revision of the article; $\mathrm{F}$ - final approval of the article
}

\section{Address for correspondence}

Dingli Xu

E-mail: dinglixu@fimmu.com

\section{Funding sources}

This study was supported by BEIJING TIDE PHARMACEUTICAL C0., LTD. (Beijing, China) but was designed and implemented independently by Guangzhou General Hospital of Guangzhou Military Command. We declare that none of the authors has any financial and personal relationships with other people or organizations that can inappropriately influence the quality of the work presented in this manuscript. There is no professional or other personal interest of any nature or kind in any product, service and/or company that may be construed as influencing the position presented in this manuscript.

Conflict of interest

None declared

Received on June 26, 2018

Reviewed on September 6, 2018

Accepted on February 18, 2019

Published online on 0ctober 22, 2019

Cite as

Duan T, Zhang J, Xiang S, Song R, Kong R, Xu D. Effectiveness and safety of intracoronary papaverine, alprostadil, and high dosages of nicorandil and adenosine triphosphate for measurement of the index of coronary microcirculatory resistance in a pig model. Adv Clin Exp Med. 2019;28(10): 1409-1418. doi:10.17219/acem/104541

DOI

10.17219/acem/104541

Copyright

Copyright by Author(s)

This is an article distributed under the terms of the Creative Commons Attribution Non-Commercial License (http://creativecommons.org/licenses/by-nc-nd/4.0/)

\begin{abstract}
Background. Papaverine is used to induce maximal hyperemia for index of coronary microcirculatory resistance (IMR) measurement in animal experiments, although it can lead to polymorphic ventricular tachycardia and ventricular fibrillation.

Objectives. This study investigated the effect of an intracoronary (IC) bolus of high adenosine triphosphate (ATP) and nicorandil doses for IMR measurement and explored the possibility of inducing maximal hyperemia with an IC alprostadil bolus.
\end{abstract}

Material and methods. Index of coronary microcirculatory resistance was measured in a hyperemic state induced by 7 experimental conditions in 21 pigs (IC bolus of papaverine (18 mg), ATP ( $40 \mu \mathrm{g}, 80 \mu \mathrm{g}, 160 \mu \mathrm{g}$, and $240 \mu \mathrm{g})$, and nicorandil ( $2 \mathrm{mg}$ and $4 \mathrm{mg})$ ). The 7 conditions were induced sequentially, and the average IMR was calculated. Because of the long-term hyperemic condition in the pilot experiments, the IMR was measured 1, 3, 5, 8, and 10 min after an IC bolus of alprostadil (10 $\mu \mathrm{g})$ in another 7 pigs.

Results. The IMR induced by $240 \mu \mathrm{g}$ of ATP or $4 \mathrm{mg}$ of nicorandil was not significantly different from that induced by $18 \mathrm{mg}$ of papaverine (both $p>0.05$ ). A strong linear correlation was observed between IMRs with papaverine $(18 \mathrm{mg})$ and nicorandil $(4 \mathrm{mg})\left(R^{2}=0.936, p<0.001\right)$ and with papaverine $(18 \mathrm{mg})$ and ATP $(240 \mu g)\left(R^{2}=0.838, p<0.05\right)$. The IC bolus of nicorandil $(4 \mathrm{mg})$ produced the smallest changes, whereas papaverine caused the most significant changes in mean blood pressure and heart rate $(p<0.05)$. Tachypnea and transient ST depression were more common with increasing ATP dosages (especially $240 \mathrm{\mu g}$ ). Alprostadil (5 min) yielded a significant hyperemic response but reduced baseline blood pressure by almost $40 \%$ for a long time.

Conclusions. Intracoronary bolus administration of $4 \mathrm{mg}$ of nicorandil was better than $18 \mathrm{mg}$ of papaverine or $240 \mu \mathrm{g}$ of ATP for induction of maximal hyperemia and IMR measurement in a pig model, whereas alprostadil was not suitable for IMR measurement.

Key words: papaverine, alprostadil, adenosine triphosphate, index of microcirculatory resistance, nicorandil 


\section{Introduction}

ST-segment elevation myocardial infarction (STEMI) usually results from acute thrombotic occlusion of a coronary artery and is the leading cause of death or loss of ability to live independently. ${ }^{1}$ The goal of reperfusion therapy with fibrinolytic drugs or primary percutaneous coronary intervention (PPCI) is to restore blood flow to ischemic areas. Although total ischemia times have improved significantly recently for patients undergoing PPCI, in-hospital mortality and heart failure have remained virtually unchanged. ${ }^{2}$ As a result, addressing coronary microvascular functional and structural obstructions, which occur frequently even after prompt epicardial recanalization of the infarct-related artery and increase the risk of cardiovascular events regardless of the epicardial disease status, is an unmet need. ${ }^{3}$

The index of coronary microcirculatory resistance (IMR) is a pressure-temperature sensor guidewire-based measurement that is performed during cardiac catherization. ${ }^{4}$ The IMR is a specific quantitative measurement used to assess coronary microvasculature function and shows a high predictive capacity for the extent and severity of myocardial infarction in patients with STEMI., ${ }^{5}$ Accurate IMR calculation requires a maximal steady state of coronary hyperemia.

Papaverine is the most commonly used pharmacological agent and the gold standard for induction of maximal hyperemia in animal experiments. ${ }^{7}$ The peak effect occurs 10-30 s after administration, with a plateau duration of approx. 45-60 s. ${ }^{8}$ However, papaverine may induce Q-T prolongation, which can lead to polymorphic ventricular tachycardia and ventricular fibrillation. ${ }^{9,10}$ In addition, an intracoronary (IC) bolus of papaverine induces a significant increase in coronary venous lactate in both animal experiments and patients with normal coronary arteries, which suggests that papaverine may produce myocardial ischemia. ${ }^{11-13}$ Adenosine triphosphate (ATP) is a precursor of adenosine that has a short half-life in plasma and is rapidly degraded into adenosine diphosphate, adenosine monophosphate and adenosine. Significant coronary vasodilation effects of ATP have been proposed to depend on its degradation to adenosine via stimulation of adenosine receptor A $2 \mathrm{a}$ and not the direct action of ATP. ${ }^{14}$ In a clinical setting, intravenous ATP $\left(140 \mu \mathrm{g} \cdot \mathrm{kg}^{-1} \cdot \mathrm{min}^{-1}\right)$ can induce a complete, true, steady-state hyperemia and enable a pressure pullback maneuver, although the optimal dosage of ATP administered via IC bolus to induce a maximal hyperemic condition is controversial ${ }^{15}$; thus, the ideal dosage of the IC ATP bolus needs to be further explored. However, no study has investigated the hyperemic effect induced by larger dosages of ATP for IMR measurement in animal experiments.

Compared with continuous intravenous infusion of adenosine for IMR measurement, administration of a IC bolus of nicorandil ( $2 \mathrm{mg}$ ) is a simple, safe and effective method to induce steady-state hyperemia for invasive physiological evaluation in a cardiac catheterization laboratory, but the effectiveness of larger dosages of nicorandil has not been reported. ${ }^{16}$ Alprostadil, which is a coronary vasodilator that acts on the microvascular system, was reported to be safe and vasoprotective in patients with pulmonary hypertension or chronic heart failure. ${ }^{17,18}$ Therefore, could alprostadil be a new agent to induce coronary hyperemia?

Due to the difficulty in studying coronary microvascular dysfunction in patients with STEMI immediately after PPCI, animal experimentation is the proper research approach. Therefore, in this study, we used papaverine as the control group in a pig model to compare the effectiveness and safety of inducing hyperemia with larger dosages of ATP and nicorandil and the feasibility and the practicality of inducing coronary hyperemia with an IC bolus of alprostadil for measurement of the IMR. Our results provide insights into the optimal administration method and dosage needed to achieve hyperemia and accurately measure the IMR in animal experiments.

\section{Material and methods}

\section{Protocol}

The Institutional Animal Care and Use Committee of Guangzhou General Hospital of Guangzhou Military Command approved the study protocol. All studies were performed in accordance with the Animal Research Reporting In Vivo Experiments (ARRIVE) guidelines for
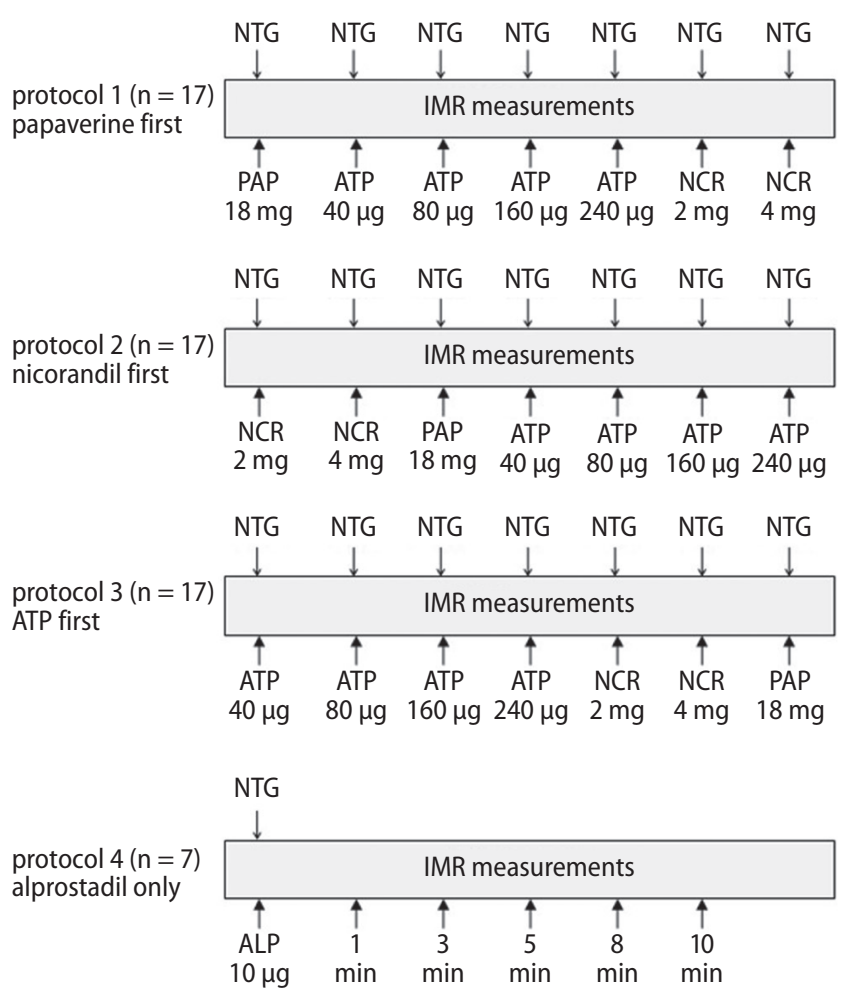

Fig. 1. Outline of the hyperemic stimuli protocol

NTG - nitroglycerine; PAP - papaverine; NCR - nicorandil; ATP - adenosine triphosphate; ALP - alprostadil; IMR - index of microcirculatory resistance. 
the reporting of animal experiments. ${ }^{19}$ The IMR was measured following induction by 7 experimental conditions in 21 pigs (IC bolus of papaverine (18 mg), ATP ( $40 \mu \mathrm{g}, 80 \mu \mathrm{g}$, $160 \mu \mathrm{g}$, and $240 \mu \mathrm{g}$ ) and nicorandil (2 mg and $4 \mathrm{mg})$ ). To exclude the possible influence of the sequence of pharmacological agents, measurements of the above 3 drugs were performed in sequential order (i.e., ATP and nicorandil were followed by papaverine in the $1^{\text {st }}$ measurement, papaverine and ATP were followed by nicorandil in the $2^{\text {nd }}$ measurement, and nicorandil and papaverine were followed by ATP in the $3^{\text {rd }}$ measurement). In addition, due to the long hyperemic condition induced in the pilot experiments, the measurement of the IMR induced by $10 \mu \mathrm{g}$ of alprostadil was measured in an additional 7 pigs. An IC bolus of nitroglycerine $(200 \mu \mathrm{g})$ was administered before each IMR measurement. Details of the experimental protocol are summarized in Fig. 1.

\section{Animal preparation}

Five days before the measurement, all 28 pigs received aspirin ( $5 \mathrm{mg} / \mathrm{kg}$ nightly), clopidogrel ( $5 \mathrm{mg} / \mathrm{kg}$ daily), perindopril (4 mg daily), and atorvastatin calcium (20 mg nightly). General anesthesia was induced by intramuscular injection of a mixture of ketamine (200 mg), Su-mian-xin (1.5 mL, mixture of haloperidol, xylidinothiazole and dihydroetorphine) and midazolam (10 mg), and then maintained with a mixture of $8 \mathrm{~mL}$ of $0.9 \%$ sodium chloride, $2 \mathrm{~mL}$ of ketamine (100 mg) and $40 \mathrm{~mL}$ of Propofol delivered continuously with a medical syringe pump through a marginal ear vein (8-18 $\mathrm{mL}$ per h). Oxygen was supplied at a rate of $2 \mathrm{~L} / \mathrm{min}$. Penicillin (4.8 million units) was injected $30 \mathrm{~min}$ before the experiment. After local injection of $10 \mathrm{~mL}$ of lidocaine in the inguinal area, the right femoral artery was exposed and isolated after skin incision and separation of subcutaneous tissue, and then a 6-French (6-F) size sheath was placed in the artery. A 6-F JR3.5 guide catheter was inserted into the left coronary artery through the arterial sheath. Then, the animals were heparinized (100 U/kg IC, with another $2500 \mathrm{U}$ added every h). Finally, a baseline angiography was performed.

\section{IMR measurement}

After calibration, a 0.014-inch-diameter coronary pressure wire (St. Jude Medical Systems, Saint Paul, USA) was advanced into the distal area of the left anterior descending coronary artery (LAD). The mean aortic pressure ( $\mathrm{Pa})$, mean distal pressure of the LAD (Pd) and mean transit time (Tmn, in seconds) of a $3 \times 3 \mathrm{~mL}$ bolus of room-temperature saline injected into the coronary artery were recorded at baseline through the 6-F guide catheter and the pressure wire. Then, a mixture of $0.9 \%$ sodium chloride and 1 hyperemic drug was injected into the left coronary artery through the 6-F JR3.5 guide catheter to induce maximal hyperemia. When the maximal steady state of coronary hyperemia was reached, a $3 \times 3 \mathrm{~mL}$ bolus of room-temperature saline was injected into the coronary artery, and the Pa, Pd and Tmn were recorded. The IMR was calculated as the mean Tmn multiplied by the Pd. The next measurement could not be made until the $\mathrm{Pa}, \mathrm{Pd}$ and heart rate recovered from the hyperemic level to the baseline level after an interval of several minutes. Seven conditions were investigated; under each condition, the IMR was measured as described above in sequential order, and the average IMR value was calculated for analyses among the 21 pigs. In addition, the time of the pressure drop from the basic to the maximal hyperemic state and the duration of the maximal hyperemic condition were recorded under every condition. The IMR induced by alprostadil $(10 \mu \mathrm{g})$ was measured in another 7 pigs due to the long hyperemic condition at $1,3,5,8$, and 10 min after the IC bolus. In all cases, a 12-lead real-time electrocardiogram (ECG) monitor system recorded the total ECG waveform, including the ventricular premature beats, ventricular tachycardia, ventricular fibrillation and ECG ST and T wave changes.

\section{Statistical analyses}

All statistical analyses were performed using SPSS v. 21.0 (IBM Corp., Armonk, USA). Categorical data was presented as a frequency or a percentage, and differences among groups were analyzed using the $\chi^{2}$ test. Continuous data was presented as medians with standard deviations (SD) or medians and interquartile ranges $\left(25^{\text {th }}-75^{\text {th }}\right)$. Differences in the IMR obtained with different hyperemic methods were analyzed using repeated measures analysis of variance (ANOVA). If the overall difference was significant, then a pairwise comparison was conducted. The Bland-Altman plot of the IMR was used to compare 2 hyperemic effects. A p-value $<0.05$ (two-sided) was considered significant.

\section{Results}

\section{Study animals}

A total of 28 pigs with an average body weight of $21.2 \pm 2.1 \mathrm{~kg}$ received IMR measurements. Twenty-one pigs were induced by papaverine $(18 \mathrm{mg})$ and different doses of ATP $(40 \mu \mathrm{g}, 80 \mu \mathrm{g}, 160 \mu \mathrm{g}$, and $240 \mu \mathrm{g})$ and nicorandil ( $2 \mathrm{mg}$ and $4 \mathrm{mg}$ ) through IC bolus administration. Conversely, 7 pigs were induced by alprostadil alone. Four pigs died during the experiment, each due to one of the following reasons: hemorrhagic shock, an anesthesia accident, left main coronary thrombosis, and reperfusion arrhythmia. A total of 51 measurements were completed for each hyperemic drug dosage when the maximal hyperemic condition was induced in 17 pigs. In another 7 pigs, the time required for the recovery of the $\mathrm{Pa}, \mathrm{Pd}$ and heart rate from the hyperemic to the baseline level after an IC bolus of alprostadil was almost $32 \mathrm{~min}$, and a significant hyperemic 
Table 1. Comparison between 4 drugs used to induce hyperemia

\begin{tabular}{|c|c|c|c|c|c|}
\hline & $\begin{array}{c}\text { Papaverine } \\
18 \mathrm{mg} \\
(\mathrm{n}=51)\end{array}$ & $\begin{array}{c}\text { ATP } \\
240 \mu g \\
(n=51)\end{array}$ & $\begin{array}{c}\text { Nicorandil } \\
4 \mathrm{mg} \\
(\mathrm{n}=51)\end{array}$ & $\begin{array}{c}\text { Alprostadil } \\
10 \mu \mathrm{g} \\
(\mathrm{n}=7)\end{array}$ & $\mathrm{p}$-value \\
\hline \multicolumn{6}{|c|}{ Baseline characteristics } \\
\hline Male [\%] & $17(80.9 \%)$ & 17 (80.9\%) & 17 (80.9\%) & $7(100 \%)$ & \\
\hline Body weight [kg] & $21.7(19.5-23.4)$ & $21.7(19.5-23.4)$ & $21.7(19.5-23.4)$ & $24.0(21.5-25.3)$ & \\
\hline Death [\%] & $4(14.3 \%)$ & $4(14.3 \%)$ & $4(14.3 \%)$ & $0(0 \%)$ & \\
\hline Baseline $\mathrm{Pa}[\mathrm{mm} \mathrm{Hg}]$ & $110(101-120)$ & $109(101-118)$ & $110(102-121)$ & $101(97-108)$ & 0.211 \\
\hline Baseline Pd [mm Hg] & $108(99-117)$ & $107(99-117)$ & $109(101-118)$ & $98(91-104)$ & 0.116 \\
\hline Baseline HR & $78(72-85)$ & $80(75-88)$ & $81(78-85)$ & $72(64-77)$ & 0.059 \\
\hline \multicolumn{6}{|c|}{ Hyperemic efficacy } \\
\hline FFR & $0.95(0.94-0.96)$ & $0.92(0.89-0.97)$ & $0.92(0.90-0.95)$ & $0.95(0.94-0.96)$ & 0.000 \\
\hline IMR & $11.5(9.8-13.1)$ & $11.6(10.0-12.9)$ & $11.3(9.2-13.7)$ & $9.6(7.2-11.5)$ & 0.213 \\
\hline CFR & $4.1(3.9-4.4)$ & $3.6(3.3-3.8)$ & $4.1(3.8-4.3)$ & $3.5(3.1-3.9)^{*}$ & 0.000 \\
\hline Time to the lowest IMR [s] & $15.7(14.0-17.0)$ & $12.8(11.0-14.0)$ & $17.7(16.0-20.0)$ & & 0.000 \\
\hline Plateau time $[\mathrm{s}]$ & $49.0(49.0-52.0)$ & $24.7(22.0-27.0)$ & $30.3(27.0-33.0)$ & & 0.000 \\
\hline $\operatorname{Tmn}[\mathrm{s}]$ & $0.16(0.13-0.19)$ & $0.15(0.13-0.17)$ & $0.14(0.11-0.16)$ & $0.15(0.12-0.16)$ & 0.005 \\
\hline \multicolumn{6}{|c|}{ Side effects } \\
\hline$\triangle \mathrm{Pa}[\mathrm{mm} \mathrm{Hg}]$ & $33(29-36)$ & $26(20-30)$ & $19(14-21)$ & $33(31-39)$ & 0.000 \\
\hline$\Delta$ Heart rate & $38(32-45)$ & $27(25-29)$ & $20(18-23)$ & $26(22-29)$ & 0.000 \\
\hline $\mathrm{R}^{* *}>20, \mathrm{n}[\%]$ & $2(11.7)$ & $11(64.7)$ & $1(5.9)$ & $7(100)$ & \\
\hline$\triangle P R$ interval [ms] & $0(0)$ & $0(0)$ & $0(0)$ & $0(0)$ & \\
\hline ST depression, n [\%] & $0(0)$ & $4(23.5)$ & $0(0)$ & $0(0)$ & \\
\hline Serious AV-nodal block, n [\%] & $0(0)$ & $0(0)$ & $0(0)$ & $0(0)$ & \\
\hline
\end{tabular}

Values given are medians (interquartile range, $25^{\text {th }}-75^{\text {th }}$ ) or rate.

- the value obtained 5 min after alprostadil administration.

* the value obtained 1 min after alprostadil administration.

${ }^{*} \mathrm{R}$ - respiratory rate; $\mathrm{Pa}$ - mean aortic pressure; $\mathrm{Pd}$ - mean distal pressure of the left anterior descending coronary artery; FFR - fractional flow reserve; IMR - index of microcirculatory resistance; CFR - coronary flow reserve; Tmn - transit mean time; $\triangle \mathrm{Pa}$ - difference in the mean aortic pressure between the maximal hyperemic condition and the baseline level; $\triangle H R$ - difference in the heart rate between the maximal hyperemic condition and the baseline level; AV - nodal block, atrioventricular block.

condition continued for nearly $10 \mathrm{~min}$; therefore, the IMR was measured $1,3,5,8$, and 10 min after alprostadil administration. The hyperemic efficacies of the 4 drugs are presented in Table 1.

\section{IMR induced by ATP}

A significant decrease was observed in the IMR with increasing ATP dosages from $40 \mu \mathrm{g}$ to $240 \mu \mathrm{g}$ (p-value for trend $<0.05)$. No significant difference was observed in the IMR induced by $240 \mu \mathrm{g}$ of ATP compared to that induced by $18 \mathrm{mg}$ of papaverine (11.6 $\pm 2.2 \mathrm{vs} 11.5 \pm 2.4$, p >0.05), but a significant difference was found in the IMRs induced by $40 \mu \mathrm{g}, 80 \mu \mathrm{g}$ and $160 \mu \mathrm{g}$ of ATP $(22.2 \pm 7.9,15.3 \pm 4.5$ and $13.2 \pm 3.3$, respectively, vs $11.5 \pm 2.4$, all $\mathrm{p}<0.05$ ). A strong linear correlation was found between the IMRs induced with the IC bolus of papaverine $(18 \mathrm{mg})$ and ATP $(240 \mu \mathrm{g})$ $\left(\mathrm{R}^{2}=0.838, \mathrm{y}=0.812 \mathrm{x}+2.219, \mathrm{p}<0.05\right)$. The agreement between the 2 sets of measurements was good, with a mean difference of 0.06 and SD of 0.44. In all measurements, the values of $1.9 \%(1 / 51)$ of the measurements were beyond the $95 \%$ confidence interval $(95 \% \mathrm{CI})$. As the ATP dosage increased, the fractional flow reserve (FFR) gradually decreased ( $p$-value for trend $<0.05$ ) and the coronary flow reserve (CFR) increased from the lowest measurement at the $40 \mu \mathrm{g}$ dosage to the highest at $240 \mu \mathrm{g}$ (p-value for trend $<0.05)$. Similar to the IMR, significant differences were found in the FFR and CFR induced by $240 \mu \mathrm{g}$ of ATP compared with those induced by papaverine (both $\mathrm{p}<0.05$ ). Under the $240 \mu \mathrm{g}$ dosage, a significant difference in the decreases in the mean arterial pressure and heart rate change were found between papaverine and ATP $(\mathrm{p}<0.05)$. Detailed results are illustrated in Fig. 2, 3 and 5.

\section{IMR induced by nicorandil}

The IMR induced by nicorandil (2 mg) was higher than that induced by papaverine (18 mg) (13.6 \pm 2.1 vs $11.5 \pm 2.4$, $\mathrm{p}<0.05)$, whereas the IMR induced by the IC bolus of nicorandil ( $4 \mathrm{mg})(11.3 \pm 2.0)$ was nearly equal to that induced by the IC bolus of papaverine (18 mg) $(11.5 \pm 2.4)$ $(p=0.999)$. A strong linear correlation was found between 


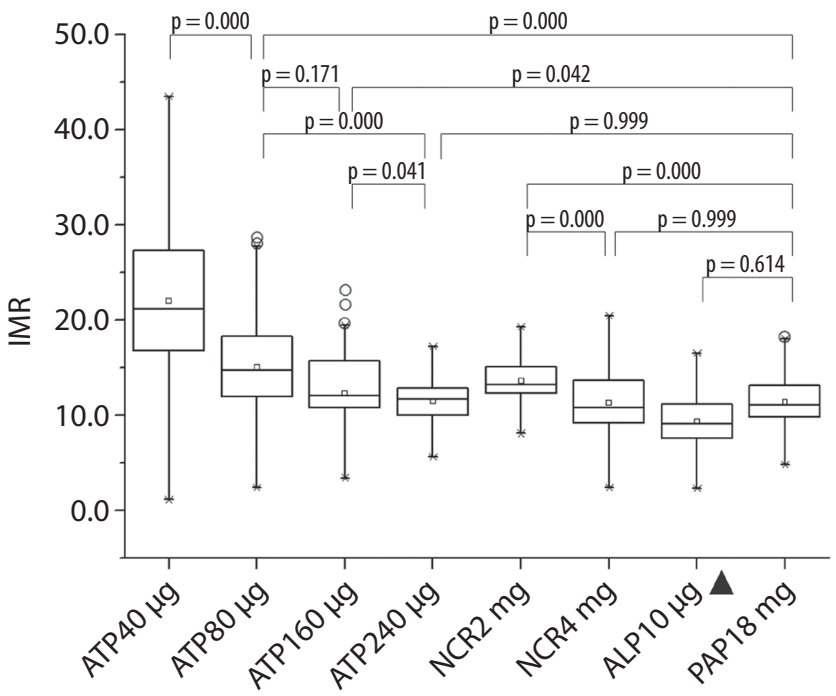

Fig. 2. Comparison of the IMR induced by an IC bolus of ATP, NCR, ALP and PAP in the non-stenotic LAD

PAP - papaverine; NCR - nicorandil; ATP - adenosine triphosphate;

ALP - alprostadil; IMR - index of microcirculatory resistance.

- The IMR value was measured under the hyperemic condition 5 min after administration of alprostadil.

the IMRs with the IC bolus of papaverine $(18 \mathrm{mg})$ and nicorandil (4 mg) ( $\left.\mathrm{R}^{2}=0.936, \mathrm{y}=1.071 \mathrm{x}-0.987, \mathrm{p}<0.001\right)$. The agreement between the 2 sets of measurements was good, with a mean difference of 0.17 and a SD of 0.45 . In all measurements, the values of $3.9 \%(2 / 51)$ of the measurements were beyond the $95 \%$ CI. A significant difference was observed in the FFR between the groups that received the IC bolus of nicorandil ( $4 \mathrm{mg}$ ) and papaverine $(18 \mathrm{mg})$ $(\mathrm{p}<0.05)$, but these differences were not found in the CFR ( $\mathrm{p}>0.05)$. Compared to the IC bolus of papaverine, fewer
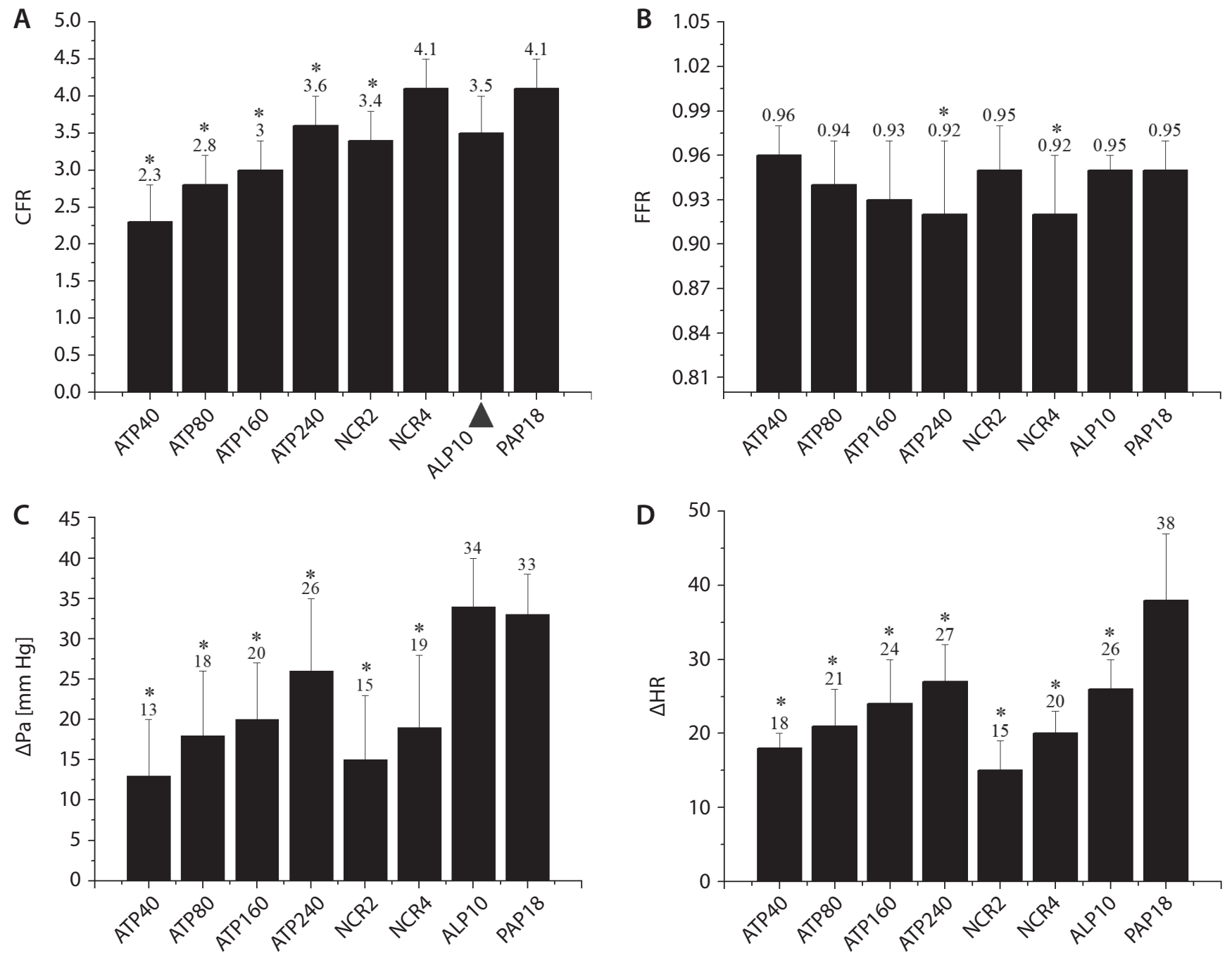

Fig. 3. Comparison of the CFR, FFR, $\triangle P$ a, and $\triangle H R$ induced by an IC bolus of ATP, NCR, ALP, and PAP in the non-stenotic left coronary artery

CFR - coronary flow reserve; FFR - fractional flow reserve; $\triangle \mathrm{Pa}$ - difference in the mean aortic pressure between the maximal hyperemic condition and the baseline level; $\triangle H R$ - difference in the heart rate between the maximal hyperemic condition and the baseline level. 


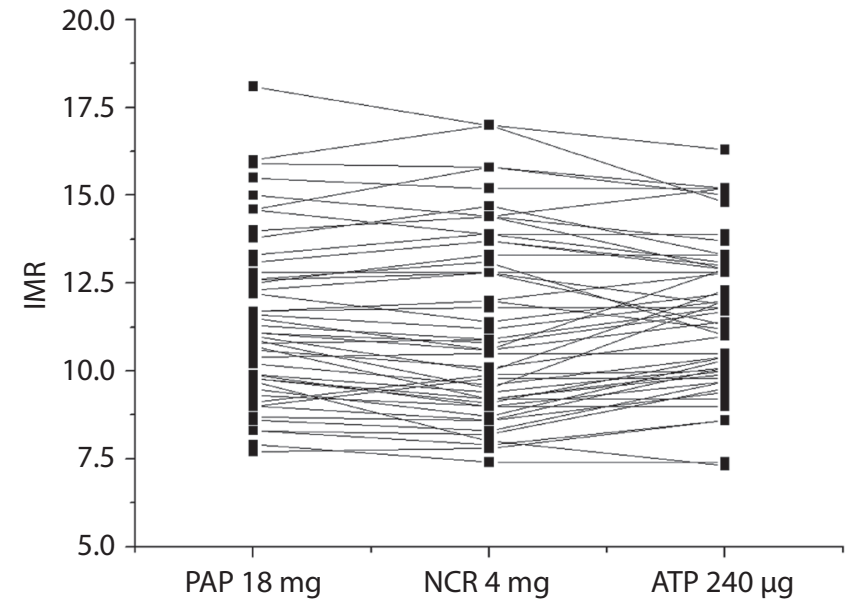

Fig. 4. Individual IMR values obtained after inducing hyperemia with papaverine (18 mg), ATP (240 $\mu \mathrm{g})$ and nicorandil (4 mg)

Abbreviations are as described in Fig. 1.

changes were detected in the mean blood pressure and heart rate in the measurements that received the IC bolus of nicorandil (both $\mathrm{p}<0.05$ ). Detailed results are illustrated in Fig. 2, 3 and 5.
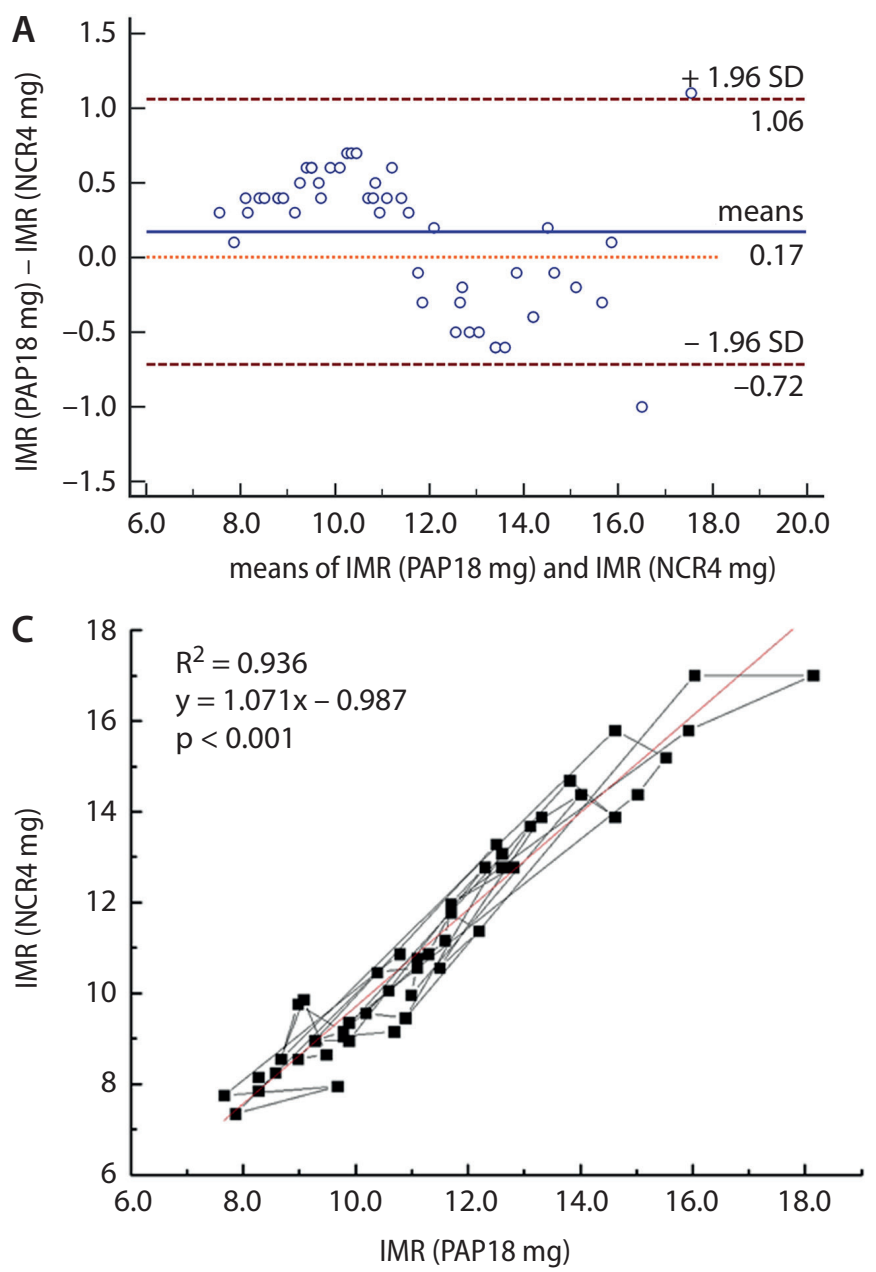

Individual IMR values among the papaverine (18 $\mathrm{mg})$, ATP $(240 \mu \mathrm{g})$ and nicorandil (4 mg) hyperemic induction groups are shown in Fig. 4.

\section{IMR induced by alprostadil}

After receiving an IC bolus of alprostadil $(10 \mu \mathrm{g})$, the IMR decreased gradually and bottomed out within 5 min before increasing slowly. Compared with papaverine, alprostadil yielded a stronger hyperemic response with a lower IMR (5 $\mathrm{min}$ ), but the difference did not reach significance $(9.6 \pm 2.2$ vs $11.5 \pm 2.4, p=0.639)$. The IC bolus of alprostadil caused large changes in the mean blood pressure, including an almost $40 \%$ reduction compared with the baseline blood pressure, and the heart rate also changed greatly. Detailed results are illustrated in Fig. 3 and 6.

\section{Side effects during induction of maximal hyperemia}

Of the 4 drugs, the IC bolus of nicorandil ( $4 \mathrm{mg}$ ) produced the smallest changes in the mean blood pressure and heart rate; in contrast, alprostadil and papaverine caused the most
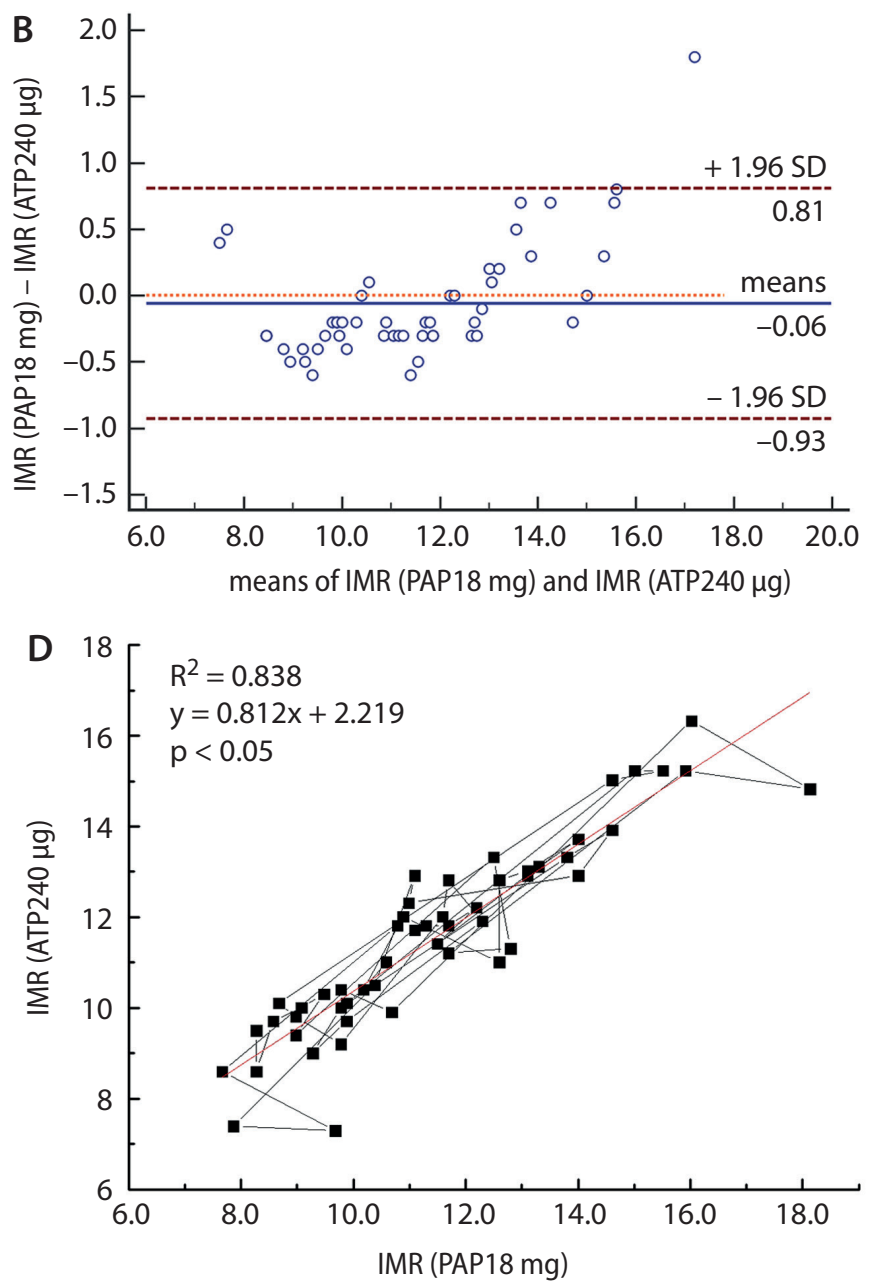

Fig. 5. Bland-Altman plots (A, B) and correlations (C, D) of IMR induced by an IC bolus of nicorandil (NCR, 4 mg), ATP (240 $\mu \mathrm{g})$ and papaverine (PAP, 18 mg) Abbreviations are as described in Fig. 1. 

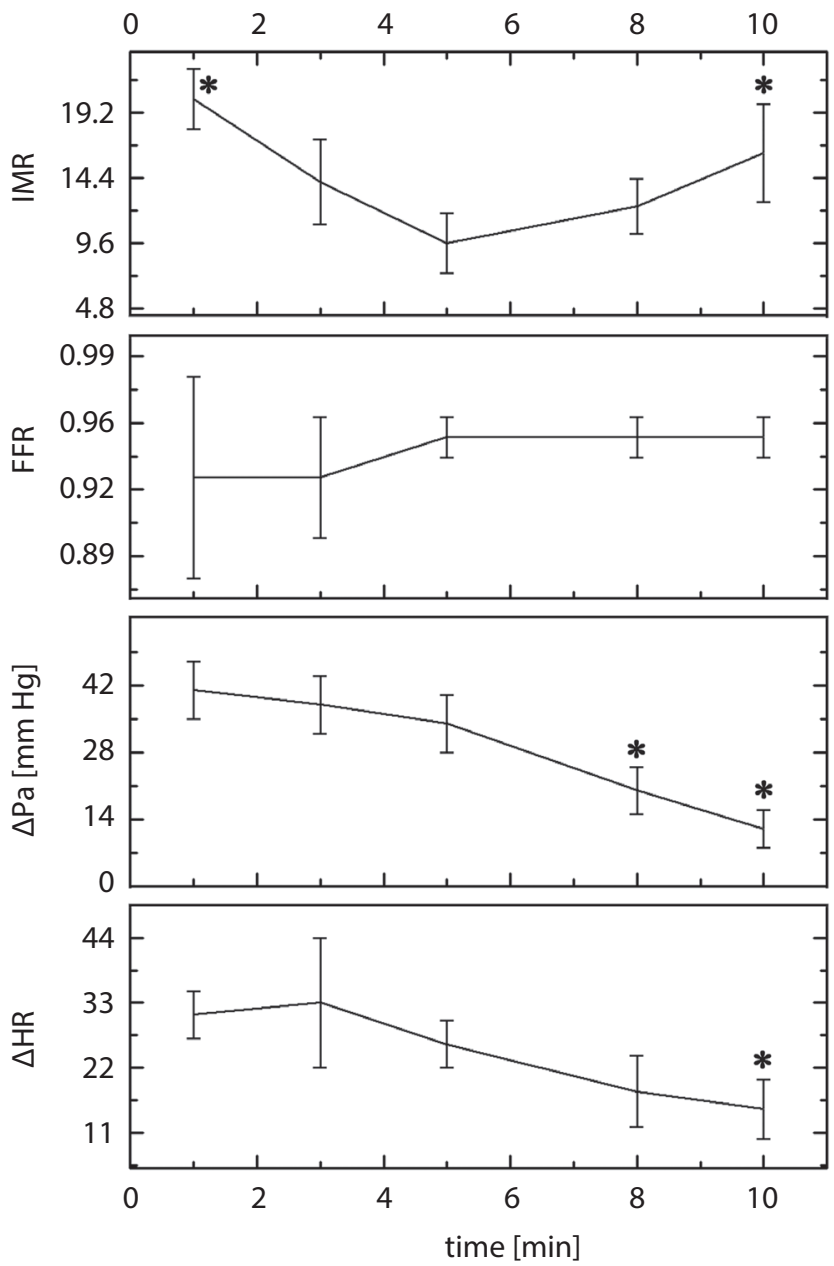

Fig. 6. Comparison of the IMR, FFR, $\triangle \mathrm{Pa}$ and $\triangle \mathrm{HR}$ under hyperemic conditions at $1,3,5,8$, and 10 min after administration of an IC bolus of alprostadil $(10 \mu \mathrm{g})$ in the non-stenotic left coronary artery

Abbreviations are as described in Fig. 1.

* Compared with the value obtained 5 min after administration of an IC bolus of alprostadil, $p<0.05$.

significant changes in the mean blood pressure and heart rate, respectively $(\mathrm{p}<0.05)$. Side effects were more common with increasing ATP dosages, especially at the $240 \mu \mathrm{g}$ dose, including tachypnea and transient ST depression. No significant changes in the PR, QRS or QT intervals on the ECG were noted, even at the highest dose administered, and no atrioventricular blocks occurred. Detailed results are illustrated in Table 1 and Fig. 3 and 6.

\section{Discussion}

In patients with STEMI, coronary microvasculature dysfunction occurs after complete reperfusion with a prevalence ranging from $5 \%$ to $50 \% .{ }^{20}$ Consistent evidence has shown that coronary microvasculature dysfunction has a strong negative impact on the prognosis, including effects on the potential benefits of percutaneous coronary intervention and an increase in the risk of early post-infarction complications, adverse left ventricular remodeling, lateonset heart failure, and mortality. ${ }^{21-23}$ Identifying a simple, reliable and convenient method to independently diagnose coronary microvasculature dysfunction is crucial for the initiation of timely treatment and effective restoration of microcirculation function. The IMR is a specific quantitative indicator for the assessment of coronary microvasculature function that is applied in most physiological assessments of myocardial blood flow but requires a maximal steady coronary hyperemic condition to achieve accurate measurement. ${ }^{3,24}$

Although perfusion of myocardial tissues has been restored in a timely manner and the condition of patients with STEMI has improved, they remain in critical condition and still need close observation to maintain life. There are many difficulties in the study of coronary microvascular dysfunction in patients with STEMI immediately after reperfusion. Therefore, investigations of the assessment methods in animal models and interventional studies of coronary microcirculation dysfunction after experimental STEMI could have great value. An ideal animal model should present with cardinal signs and pathology that resemble the human disease. Quality preclinical data offers valuable insights for translational research. Pigs resemble humans in the context of minimal preexisting coronary collaterals and similar coronary anatomy and physiology and, therefore, are commonly used in animal experiments of myocardial infarction. ${ }^{25}$ In this study, we compared the hyperemic effects of different pharmaceutical agents on IMR measurements using a pig model.

As a precursor of adenosine, ATP is equivalent to adenosine for both CFR and FFR measurements. The current recommendation for IC ATP dosing is $60 \mu \mathrm{g}$ in the left coronary artery; if necessary, the dosage can be increased incrementally by $30 \mu \mathrm{g}$ to a maximum of $150 \mu \mathrm{g} .{ }^{26} \mathrm{How}-$ ever, the ideal dosage of the IC route is a controversial issue that has been addressed in several clinical reports. ${ }^{27-30}$

According to previous reports ${ }^{14,15}$ that used $18 \mathrm{mg}$ of papaverine as the control group, we compared the differences in the IMRs induced by $40 \mu \mathrm{g}, 80 \mu \mathrm{g}, 160 \mu \mathrm{g}$, and $240 \mu \mathrm{g}$ of ATP via the left coronary artery. A significant dosedependent relationship was observed with hyperemia, with the higher dosage yielding a better hyperemic response. The higher dosage of ATP resulted in a significantly lower IMR than the lower dosages. The lowest IMR was induced by $240 \mu \mathrm{g}$ of ATP, and the highest was induced by $40 \mu \mathrm{g}$ of ATP. Based on the IMR measured following IC infusion of $240 \mu \mathrm{g}$ of ATP, this condition was the only experimental condition that generated hyperemia comparable to that obtained with $18 \mathrm{mg}$ of papaverine, whereas $40 \mu \mathrm{g}, 80 \mu \mathrm{g}$ and $160 \mu \mathrm{g}$ of ATP did not induce comparable states. Meanwhile, significant differences in the IMR were induced by the 4 ATP dosages. No difference in the IMR was found between the groups administered the IC bolus of ATP $(240 \mu \mathrm{g})$ and papaverine $(18 \mathrm{mg})$; and a strong linear correlation existed between the IMRs induced with the IC bolus 
of papaverine $(18 \mathrm{mg})$ and ATP $(240 \mu \mathrm{g})$, although only 1 IMR value was beyond the $95 \%$ conformance scope. This result indicated that the hyperemic effects on the IMR induced by the 2 agents were equivalent. Similar to the IMR, significant differences were found in the FFR and CFR induced by $240 \mu \mathrm{g}$ of ATP compared with the effects induced by papaverine. These results showed that the current recommended dosage of IC ATP is not sufficient in animal experiments; thus, a higher dosage is essential to ensure an adequate hyperemic response. This conclusion is supported by several studies. Jeremias et al. compared the effect of ATP on the measurement of the CFR in 6 healthy mongrel dogs. Their results showed a dose-dependent relationship of the CFR with IC administration of ATP via the non-stenotic left circumflex coronary artery, with the largest value induced by the maximal dosage.${ }^{27}$ Casella et al. suggested that an IC bolus dosage up to $150 \mu \mathrm{g}$ should be better; for the standard doses, $10 \%$ measurement values may be underestimated. ${ }^{28}$ Leone et al. demonstrated that only much higher doses $(600 \mu \mathrm{g})$ produced real hyperemic efficacy ${ }^{29}$ Lower doses of the drug are associated with an inferior diagnostic accuracy, and thus, a higher dosage may be required.

The FFR dropped slightly as the ATP dosage increased. The mean FFR values were very similar under all experimental conditions, and the difference between the highest and lowest values was only 0.04 . This finding also differed from those of some previous studies on epicardial coronary stenosis, ${ }^{31,32}$ including angiographically intermediate stenosis, multivessel disease, and sequential stenosis. The discrepancy could result from the epicardial arteries being normal in our study. This finding indicated that the effects of different administration dosages on the normal epicardial coronary artery were similar. Unlike the FFR, the CFR increased slightly as the ATP dosage increased, and significant differences in the CFR were observed between the groups that received the IC bolus of ATP $(40 \mu \mathrm{g}$, $80 \mu \mathrm{g}$ and $160 \mu \mathrm{g})$ and papaverine $(18 \mathrm{mg})$.

Due to opening of ATP-sensitive potassium channels in coronary resistant arterioles and its possession of a nitrate moiety effect, nicorandil has been used as an antianginal drug for several years. ${ }^{33-35}$ Recently, nicorandil has been used for invasive physiological assessments as a novel hyperemic agent in the cardiac catheterization laboratory. ${ }^{36,37}$ In our study, $2 \mathrm{mg}$ of nicorandil yielded a weaker hyperemic response with a higher IMR than $4 \mathrm{mg}$ of nicorandil or $18 \mathrm{mg}$ of papaverine, indicating that maximal hyperemia in the animal body was not achieved by lower dosages. No difference in the IMR was induced between the IC bolus of nicorandil (4 $\mathrm{mg}$ ) and papaverine $(18 \mathrm{mg})$, and a strong linear correlation existed between the IMRs obtained with the IC bolus of papaverine $(18 \mathrm{mg})$ and nicorandil ( $4 \mathrm{mg}$ ), with only 2 IMR values beyond the $95 \%$ conformance scope. This finding indicated that the hyperemic effects on the IMRs induced by the 2 agents were equivalent. The FFR value induced by $4 \mathrm{mg}$ of nicorandil was much lower than that of papaverine, but no significant difference in the CFR was observed.

Our results showed that the effect of nicorandil on inducing maximal hyperemia appeared to contradict the results of some previous studies. Jang et al. compared the effect of nicorandil on the measurement of the IMR and FFR with adenosine as the clinical agent. ${ }^{16}$ The study suggested that the hyperemic effect of an IC bolus of $2 \mathrm{mg}$ was equal to that of the IV standard dosage of adenosine and was superior to the effect of an IC bolus of adenosine $(80 \mu \mathrm{g})$, Tanaka et al. reported that no further decrease occurred in the FFR at nicorandil doses over $2 \mathrm{mg}^{38}$

Several factors may explain the differences between the clinical setting and the experimental results, such as intrinsic differences in coronary anatomy, differences in drug metabolism or different heart sizes between pigs and humans. Some studies have reported that despite the similarity of the coronary arterial system with respect to morphology and size and even the capillary diameter between pigs and humans, slight differences exist. ${ }^{25,39}$ In addition, in our study, we used IMR instead of FFR or CFR as the main observation index, which was different from previous studies. The IMR is a reliable measurement that has been specifically dedicated to assessing coronary microvascular dysfunction, and changes in the IMR reflect fluctuations of the microcirculation condition induced by hyperemic agents in the same vessel. Conversely, as a modification of the translesional pressure gradient, the FFR detects clinically relevant lumen narrowing of the epicardial coronary artery.

The IC bolus of nicorandil $(4 \mathrm{mg})$ and ATP $(240 \mu \mathrm{g})$ were both slower in reaching maximal hyperemia than papaverine. However, the hyperemic duration was longer than that obtained with an IC bolus of papaverine. The peak effect time of maximal coronary hyperemia for ATP $(240 \mu \mathrm{g})$ and nicorandil ( $4 \mathrm{mg}$ ) lasted $25 \mathrm{~s}$, whereas the peak effect time of papaverine was $20 \mathrm{~s}$. In our study, an average of $4 \mathrm{~s}$ was required to finish injecting a $1 \times 3 \mathrm{~mL}$ bolus of room-temperature saline into the coronary artery, and thus, the peak effect time was adequate for experienced hands (but not new learners) to conduct measurements of a $3 \times 3 \mathrm{~mL}$ bolus of saline during the experiments.

Alprostadil is a potent systemic vasodilator with important endogenous flow-regulating activity to maintain blood flow to vital organs. ${ }^{40}$ This study is the first to evaluate the coronary hyperemic effect of alprostadil via IC bolus in IMR measurements in pigs. After administration of an IC bolus of alprostadil, the systemic blood pressure decreased immediately; the fastest rate reduction was nearly $30-40 \%$ in the first $2 \mathrm{~min}$, and the significantly lower pressure lasted for more than $10 \mathrm{~min}$. Thirty-two minutes later, the systemic blood pressure recovered from the hyperemic condition to the baseline level. As a result, IMR measurements were performed in an additional 7 pigs at 1, 3, 5, 8, and $10 \mathrm{~min}$ after the IC bolus. The maximal hyperemic effect of alprostadil occurred 5 min after administration. Alprostadil produced a lower IMR than papaverine, 
but the difference in the IMR was not significant between the 2 drugs. The significant disadvantages of an IC bolus of alprostadil were drastic fluctuation of blood pressure and the long hyperemic duration time, which might influence vital organ perfusion. Thus, administration of an IC bolus of alprostadil is not suitable to produce a hyperemic response for IMR measurement.

During the experiments, blood pressure and heart rate were the most vulnerable measurements. Alprostadil caused the most severe decrease in the mean blood pressure, and papaverine led to the largest increase in the heart rate and decrease in the mean blood pressure. Conversely, an IC bolus of nicorandil ( $4 \mathrm{mg}$ ) produced the smallest changes in the mean blood pressure and heart rate, whereas the influence of ATP was at the mid-level of the 4 pharmaceutical agents. The most notable side effect of IC administration of ATP was a slight increase in tachypnea and transient ST depression. We did not observe any atrioventricular block or other changes in the PR and QRS intervals on the ECG, even at the highest dose. Therefore, as a safe and effective agent, $4 \mathrm{mg}$ of nicorandil seems to be useful in animals with lower blood pressure, and $240 \mu \mathrm{g}$ of ATP is a safe and valid agent to induce hyperemia via IC bolus administration, although it produced more side effects than nicorandil.

The study has several limitations that need to be addressed. Firstly, the dose-response relationship could be further confirmed by including other dosages of ATP, such as $180 \mu \mathrm{g}, 200 \mu \mathrm{g}$ or $220 \mu \mathrm{g}$. Secondly, we investigated only the hyperemic state in the left coronary artery in this study and did not examine that of the right coronary artery. Future studies will be required to confirm our results in the right coronary artery. Thirdly, the left coronary arteries of pigs were normal in this experiment without angiographic stenosis (i.e., 40-70\%). Whether the results of normal arteries are same as those obtained with arteries with lesions is unclear.

\section{Conclusions}

In conclusion, both ATP and nicorandil are safe drugs that can achieve coronary hyperemia for the measurement of IMR in the left coronary artery; importantly, only higher dosages $(240 \mu \mathrm{g}$ of ATP or $4 \mathrm{mg}$ of nicorandil) produced a hyperemic condition equivalent to papaverine in animal experiments, and nicorandil had fewer side effects than papaverine and ATP. Alprostadil is not suitable for inducing maximal hyperemia due to the severe decrease in blood pressure.

\section{References}

1. Li J, Li X, Wang Q, et al. ST-segment elevation myocardial infarction in China from 2001 to 2011 (the China PEACE-Retrospective Acute Myocardial Infarction Study): A retrospective analysis of hospital data. Lancet. 2015;385(9966):441-451.

2. Menees DS, Peterson ED, Wang Y, et al. Door-to-balloon time and mortality among patients undergoing primary $\mathrm{PCl}$. N Eng J Med. 2013;369(10):901-909.
3. Niccoli G, Scalone G, Lerman A, Crea F. Coronary microvascular obstruction in acute myocardial infarction. Eur Heart J. 2016;37(13): 1024-1033.

4. Fearon $W$, Balsam $L$, Farouque $H$, et al. Novel index for invasively assessing the coronary microcirculation. Circulation. 2003;107(25): 3129-3132.

5. McGeoch R, Watkins S, Berry C, et al. The index of microcirculatory resistance measured acutely predicts the extent and severity of myocardial infarction in patients with st-segment elevation myocardial infarction. JACC Cardiovasc Interv. 2010;3(7):715-722.

6. Cuculi F, De Maria G, Meier P, et al. Impact of microvascular obstruction on the assessment of coronary flow reserve, index of microcirculatory resistance, and fractional flow reserve after ST-segment elevation myocardial infarction. J Am Coll Cardiol. 2014;64(18): 1894-1904.

7. Vrolix M, Piessens J, De Geest H. Torsades de pointes after intracoronary papaverine. Eur Heart J. 1991;12(2):273-276.

8. van der Voort PH, van Hagen E, Hendrix G, van Gelder B, Bech JW, Pijls NH. Comparison of intravenous adenosine to intracoronary papaverine for calculation of pressure-derived fractional flow reserve. Cathet Cardiovasc Diagn. 1996;39(2):120-125.

9. Wilson RF, White CW. Serious ventricular dysrhythmias after intracoronary papaverine. Am J Cardiol. 1988;62(17):1301-1302.

10. Kern MJ, Deligonul U, Serota H, Gudipati C, Buckingham T. Ventricular arrhythmia due to intracoronary papaverine: Analysis of QT intervals and coronary vasodilatory reserve. Cathet Cardiovasc Diagn. 1990;19(4):229-236.

11. Christensen CW, Rosen LB, Gal RA, Haseeb M, Lassar TA, Port SC. Coronary vasodilator reserve. Comparison of the effects of papaverine and adenosine on coronary flow, ventricular function, and myocardial metabolism. Circulation. 1991;83(1):294-303.

12. Takeuchi M, Nohtomi Y, Kuroiwa A. Intracoronary papaverine induced myocardial lactate production in patients with angiographically normal coronary arteries. Cathet Cardiovasc Diagn. 1996;39(2):126-130.

13. Egashira K, Inou T, Hirooka Y, Yamada A, Urabe Y, Takeshita A. Evidence of impaired endothelium-dependent coronary vasodilatation in patients with angina pectoris and normal coronary angiograms. N Engl J Med. 1993;328(23):1659-1664.

14. De Bruyne B, Pijls NH, Barbato E, et al. Intracoronary and intravenous adenosine 5 '-triphosphate, adenosine, papaverine, and contrast medium to assess fractional flow reserve in humans. Circulation. 2003;107(14):1877-1883.

15. Layland J, Carrick D, Lee M, Oldroyd K, Berry C. Adenosine: Physiology, pharmacology, and clinical applications. JACC Cardiovasc Interv. 2014;7(6):581-591.

16. Jang $H$, Koo B, Lee $H$, et al. Safety and efficacy of a novel hyperaemic agent, intracoronary nicorandil, for invasive physiological assessments in the cardiac catheterization laboratory. Eur Heart J. 2013; 34(27):2055-2062.

17. Gupta V, Rawat A, Ahsan F. Feasibility study of aerosolized prostaglandin E1 microspheres as a noninvasive therapy for pulmonary arterial hypertension. J Pharm Sci. 2010;99(10):1774-1789.

18. Hülsmann M, Stefenelli T, Berger R, et al. Response of right ventricular function to prostaglandin e1 infusion predicts outcome for severe chronic heart failure patients awaiting urgent transplantation. J Heart Lung Transplant. 2000;19(10):939-945.

19. Kilkenny C, Browne W, Cuthill I, Emerson M, Altman D. Improving bioscience research reporting the ARRIVE guidelines for reporting animal research. PLoS Biol. 2010;8(6):e1000412.

20. Niccoli G, Burzotta F, Galiuto L, Crea F. Myocardial no-reflow in humans. J Am Coll Cardiol. 2009;54(4):281-292.

21. Fearon W, Shah M, $\mathrm{Ng} \mathrm{M}$, et al. Predictive value of the index of microcirculatory resistance in patients with st-segment elevation myocardial infarction. J Am Coll Cardiol. 2008;51(5):560-565.

22. Ndrepepa G, Tiroch K, Fusaro M, et al. 5-year prognostic value of noreflow phenomenon after percutaneous coronary intervention in patients with acute myocardial infarction. J Am Coll Cardiol. 2010;55(21): 2383-2389.

23. Hamirani Y, Wong A, Kramer C, Salerno M. Effect of microvascular obstruction and intramyocardial hemorrhage by CMR on LV remodeling and outcomes after myocardial infarction: A systematic review and meta-analysis. JACC Cardiovasc Imaging. 2014;7(9):940-952. 
24. Ng M, Yeung A, Fearon W. Invasive assessment of the coronary microcirculation: superior reproducibility and less hemodynamic dependence of index of microcirculatory resistance compared with coronary flow reserve. Circulation. 2006;113(17):2054-2061.

25. Kumar M, Kasala ER, Bodduluru LN, et al. Animal models of myocardial infarction: Mainstay in clinical translation. Regul Toxicol Pharmacol. 2016;76:221-230.

26. Kern MJ, Lerman A, Bech JW, et al; American Heart Association Committee on Diagnostic and Interventional Cardiac Catheterization, Council on Clinical Cardiology. Physiological assessment of coronary artery disease in the cardiac catheterization laboratory: A scientific statement from the American Heart Association Committee on Diagnostic and Interventional Cardiac Catheterization, Council on Clinical Cardiology. Circulation. 2006;114(12):1321-1341.

27. Jeremias A, Filardo S, Whitbourn R, et al. Effects of intravenous and intracoronary adenosine 5 '-triphosphate as compared with adenosine on coronary flow and pressure dynamics. Circulation. 2000;101(3): 318-323.

28. Casella G, Leibig M, Schiele TM, et al. Are high doses of intracoronary adenosine an alternative to standard Intravenous adenosine for the assessment of fractional flow reserve? Am Heart J. 2004;148(4): 590-595.

29. Leone AM, Porto I, De Caterina A, et al. Maximal hyperemia in the assessment of fractional flow reserve: intracoronary adenosine versus intracoronary sodium nitroprusside versus intravenous adenosine: The NASCI (Nitroprussiato versus Adenosina nelle Stenosi Coronariche Intermedie) study. JACC Cardiovasc Interv. 2012;5(4):402-408.

30. De Luca G, Venegoni L, Iorio S, Giuliani L, Marino P. Effects of increasing doses of intracoronary adenosine on the assessment of fractional flow reserve. JACC Cardiovasc Interv. 2011;4(10):1079-1084.
31. Pijls N, Sels J. Functional measurement of coronary stenosis. J Am Coll Cardiol. 2012;59(12):1045-1057.

32. Drenjancevic I, Koller A, Selthofer-Relatic K, Grizelj I, Cavka A. Assessment of coronary hemodynamics and vascular function. Prog Cardiovasc Dis. 2015;57(5):423-430.

33. Markham A, Plosker G, Goa KL. Nicorandil: An updated review of its use in ischemic heart disease with emphasis on its cardioprotective effects. Drugs. 2000;60(4):955-974.

34. Miyazawa A, Ikari Y, Tanabe $K$, et al. Intracoronary nicorandil prior to reperfusion in acute myocardial infarction. Eurolntervention. 2006; 2(2):211-217.

35. Hirohata A, Yamamoto K, Hirose E, et al. Nicorandil prevents microvascular dysfunction resulting from $\mathrm{PCl}$ in patients with stable angina pectoris: A randomised study. Eurolntervention. 2014;9(9):1050-1056.

36. Lee J, Kato D, Oi M, et al. Safety and efficacy of intracoronary nicorandil as hyperaemic agent for invasive physiological assessment: A patient-level pooled analysis. Eurolntervention. 2016;12(2):e208-215.

37. Kobayashi $\mathrm{Y}$, Okura $\mathrm{H}$, Neishi $\mathrm{Y}$, et al. Additive value of nicorandil on ATP for further inducing hyperemia in patients with an intermediate coronary artery stenosis. Coron Artery Dis. 2017;28(2):104-109.

38. Tanaka N, Takahashi $Y$, Ishihara $\mathrm{H}$, Kawakami $\mathrm{T}$, Ono H. Usefulness and safety of intracoronary administration of nicorandil for evaluating fractional flow reserve in Japanese patients. Clin Cardiol. 2015;38(1):20-24.

39. Pijls N, van Son J, Kirkeeide R, De Bruyne B, Gould K. Experimental basis of determining maximum coronary, myocardial, and collateral blood flow by pressure measurements for assessing functional stenosis severity before and after percutaneous transluminal coronary angioplasty. Circulation. 1993;87(4):1354-1367.

40. Miller SB. Prostaglandins in health and disease: An overview. Semin Arthritis Rheum. 2006;36(1):37-49. 